\title{
Low Temperature Crystallization of TiNi Films by Ion Irradiation
}

\author{
N. Ikenaga ${ }^{\mathrm{a}, 1}, \mathrm{Y}_{\text {Kishi }}{ }^{1}$, Z. Yajima $^{1}$ and N. Sakudo ${ }^{1}$ \\ ${ }^{1}$ Research Laboratory for Integrated Technological Systems, Kanazawa Institute of Technology, 3-1 Yatsukaho, \\ Hakusan, Ishikawa 924-0838, Japan
}

\begin{abstract}
TiNi films are well known as a typical shape memory alloy (SMA), and are expected to be promising materials for micro actuators. The film crystallization needed for making the shape memory property appear had been usually realized by high temperature (above $450^{\circ} \mathrm{C}$ ) annealing process during and/or after the sputtering deposition. As a special case, it was reported that the crystallization temperature could be lowered by enhancing the energies of sputtered particles with a single-target sputtering deposition. However, this method could not be applied to the multitarget sputtering deposition, which was essential to precisely controlling the TiNi film composition. Furthermore, it also had a difficulty in freely controlling the energies of sputtered particles. In order to resolve the problem we developed a new apparatus as well as a new process for lowering the crystallization temperature by using ion irradiation, and realized the crystallization at $200{ }^{\circ} \mathrm{C}$ of substrate temperature. The details of the film characteristics were reported in another paper. In order to further lower the crystallization temperature we try to improve the biasvoltage introduction into vacuum as well as to optimize the ion irradiation conditions. Resultantly, we have realized the low temperature crystallization at $150{ }^{\circ} \mathrm{C}$ of substrate temperature.
\end{abstract}

\section{Introduction}

TiNi shape memory alloy (SMA) films are expected to be promising materials for micro actuators in micro electro-mechanical systems (MEMS). Their work outputs per volume exceed those of other ferroic materials like PZT. Since the shape memory effect arises from a martensitic phase transformation between the hightemperature B2 phase and the low-temperature B19 (martensite) phase, the film has to be crystallized in order to make the shape memory property appear. Until sometime ago the crystallization of sputtering-deposited TiNi film had been usually realized by high temperature (above $450{ }^{\circ} \mathrm{C}$ ) annealing process during and/or after the deposition [1,2,3]. As a special case, it was reported that the crystallization temperature could be lowered by utilizing high-energy sputtered particles with a single-target sputtering deposition [4]. However, this method could not be applied to the multi-target sputtering deposition, the employment of which was considered essential to precisely controlling the TiNi film composition. Furthermore, it also had a difficulty in freely controlling the energies of sputtered particles, which played the important role in lowering the crystallization temperature. On the other hand, the TiNi film composition was an important factor to determine the martensitic phasetransformation temperature $[3,5,6]$. We reported that the TiNi film composition could be precisely controlled with a multi-target RF magnetron sputtering deposition by separately determining the sputtering condition for each target. At the same time a preliminary experiment implying the possibility to lower the crystallization temperature by simultaneous ion irradiation during deposition was reported, too [7]. Afterward we could lower the crystallization temperature down to $200^{\circ} \mathrm{C}$ by optimizing the deposition conditions as well as the ion irradiation conditions. The details of the film characteristics are shown in another paper [8]. In this paper, we try to further lower the crystallization temperature by improving the bias-voltage introduction as well as optimizing the ion irradiation conditions. The ion energy is controlled by changing the pulse bias voltage applied to the substrate, although the ion energy expressed in $\mathrm{eV}$ is not exactly equal to the pulse bias voltage in $\mathrm{V}$. It is difficult to get the exact ion energy from the pulse bias voltage, because the plasma potential of the ICP for ion irradiation also changes itself depending on the pulse bias voltage. On the other hand the ion irradiation rate is controlled by changing the plasma density. The deposited TiNi films are examined with an X-ray diffraction (XRD) and an energy dispersive X-ray spectrometer (EDS). We discuss the effects of ion irradiation on the crystallization temperature of TiNi film.

\footnotetext{
a e-mail: n-ikenaga@neptune.kanazawa-it.ac.jp
} 


\section{Experimental}

The TiNi films were deposited on Si substrates with a multi-target RF magnetron sputtering deposition system which was equipped with an ion irradiation system as shown in Fig.1. The deposition system mainly consisted of four separate confocal targets as well as a temperature-controlled substrate holder of $101 \mathrm{~mm}$ in diameter. However, the specific difference of this apparatus from traditional sputtering deposition systems is that an ICP (inductively coupled plasma) source is installed between a set of the targets and the substrate. The plasma was generated by an RF power independent of the RF powers for sputter sources. Ar ions which were generated by the ICP source with a one-turn antenna of $150 \mathrm{~mm}$ in diameter were accelerated to the substrate by applying pulse bias voltages to the substrate holder. However, the ion energy expressed in eV was not exactly equal to the pulse bias voltage in $\mathrm{V}$ as mentioned above. In order to suppress the plasma-potential variation as much as possible we coated the pulse bias voltage feeder in vacuum to the substrate holder with ceramics.

In order to realize the film composition of around $50 \%$ of Ti/Ni ratio we used three targets of $50 \mathrm{~mm}$ in diameter, two of $\mathrm{Ti}$ ( $99.9 \%$ in purity) and one of $\mathrm{Ni}$ ( $99.9 \%$ in purity), since the ion sputtering ratio of Ni was roughly two times that of Ti. The films of $1 \mu \mathrm{m}$ in thickness were deposited on $\mathrm{Si}(001)$ substrates of $10 \mathrm{~mm} \times 10 \mathrm{~mm}$ in area at a substrate temperature from $200{ }^{\circ} \mathrm{C}$ to $500{ }^{\circ} \mathrm{C}$. Ar gas pressure was set at $0.1 \mathrm{~Pa}$ by adjusting the gas flow as well as the conductance valve. The target-substrate distance was $150 \mathrm{~mm}$. Since the Ti deposition rate per minute $\left(\mathrm{DR}_{\mathrm{Ti}}\right)$ and the $\mathrm{Ni}$ deposition rate $\left(\mathrm{DR}_{\mathrm{Ni}}\right)$ were separately controlled by adjusting the RF power for each target, the ratio of $\mathrm{DR}_{\mathrm{Ti}}$ to $\mathrm{DR}_{\mathrm{N}} \mathrm{i}$ was freely selected from 1.3 to 1.6 , while keeping the total deposition rate for the TiNi film at $10 \mathrm{~nm} / \mathrm{min}$. Resultantly, we found the substrate pulse bias voltage of around $-60 \mathrm{~V}$ seemed optimum to lower the crystallization temperature.

The film thickness was measured with a surface profilometry (Dektak3, ULVAC) from the step height at the edge of masked part of $\mathrm{Si}$ substrate. The film composition was evaluated with energy dispersive X-ray spectroscopy (EDS; EMAX-5770, HORIBA) operated at 20keV. The amount of each elemental composition was averaged with EDS data from three points within a surface area of $\phi 1 \mathrm{~mm}$ on Si substrate. An X-ray powder diffraction (XRD; RAD-2R, RIGAKU) with $\mathrm{Cu} \mathrm{K \alpha}(\lambda=1.54056 \AA)$ line was used for the measurement of the degree of crystallization. The plasma density in ICP was measured for each plasma condition with double probe measurement system (L2P, JE Plasma Consult GMbH).

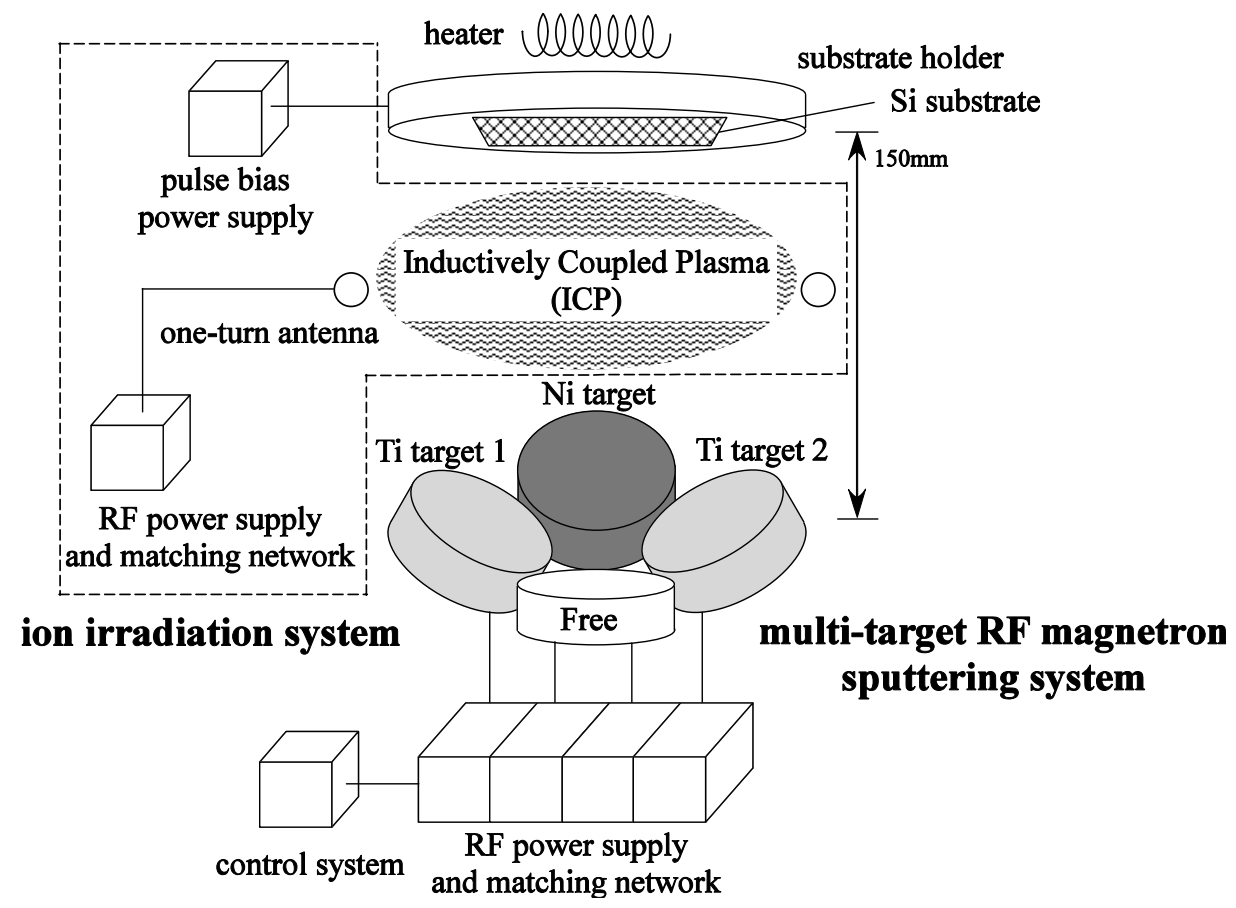

Fig. 1. Schematic illustration of a multi-target RF magnetron sputtering system equipped with an ion irradiation system.

\section{Results and discussion}

The ion irradiation and sputtering deposition parameters are shown in Table 1. Ni compositions of the deposited films under these conditions were between 50.5 at. \% and 51.5 at. \%. Fig. 2 shows the XRD profiles of (a) samples MMSD-1, 2 and (b) samples MMSDI-3, 4. Samples MMSD-1 and 2 were made without ion irradiation 
at $500{ }^{\circ} \mathrm{C}$ and $400{ }^{\circ} \mathrm{C}$ of substrate temperature, respectively. The TiNi film deposited at $400{ }^{\circ} \mathrm{C}$ was not crystallized. On the other hand, both samples MMSDI-3 and 4, which were deposited at $400{ }^{\circ} \mathrm{C}$ and $200{ }^{\circ} \mathrm{C}$ of substrate temperature by using simultaneous ion irradiation, were crystallized. These results show that the simultaneous ion irradiation during deposition is efficient in lowering the crystallization temperature of sputtering-deposited TiNi film. But the spectrum peak from the ion-irradiated sample is broader. It is considered that the peak broadening is attributable to the strain in TiNi structure, which was caused by ion irradiation. This also suggests that the crystal structures, i.e. grain size, orientation etc., would be different from those of the crystallized sample without ion irradiation (sample MMSD-1).

Table 1. Ion irradiation and sputtering deposition parameters

\begin{tabular}{|c|c|c|c|c|c|c|c|c|}
\hline $\begin{array}{l}\text { Sample } \\
\text { number }\end{array}$ & $\begin{array}{l}\text { Substrate } \\
\text { temperature } \\
\left({ }^{\circ} \mathrm{C}\right)\end{array}$ & $\begin{array}{l}\text { Pulse bias } \\
\text { voltage } \\
\text { (V) }\end{array}$ & $\begin{array}{l}\text { Pulse bias } \\
\text { repetition } \\
\text { frequency } \\
(\mathrm{Hz})\end{array}$ & $\begin{array}{l}\text { Pulse bias } \\
\text { duty ratio } \\
(\%)\end{array}$ & $\begin{array}{l}\text { RF power for } \\
\text { ICP } \\
(\mathrm{W})\end{array}$ & $\begin{array}{l}\text { Ar pressure } \\
(\mathrm{Pa})\end{array}$ & $\begin{array}{l}\text { RF power } \\
\text { for Ni target } \\
\text { (W) }\end{array}$ & $\begin{array}{l}\text { RF power } \\
\text { for Ti1 and } \\
\text { Ti2 targets } \\
\text { (W) }\end{array}$ \\
\hline MMSD-1 & 500 & 0 & \multirow{2}{*}{-} & \multirow{2}{*}{ - } & 0 & \multirow{10}{*}{0.1} & \multirow{10}{*}{100} & \multirow{10}{*}{200} \\
\hline MMSD-2 & 400 & 0 & & & 0 & & & \\
\hline MMSDI-3 & 400 & -60 & \multirow{8}{*}{$1 \mathrm{k}$} & \multirow{8}{*}{20} & 500 & & & \\
\hline MMSDI-4 & 200 & -60 & & & 500 & & & \\
\hline MMSDI-5 & 200 & -60 & & & 200 & & & \\
\hline MMSDI-6 & 200 & -60 & & & 100 & & & \\
\hline MMSDI-7 & 200 & -60 & & & 50 & & & \\
\hline MMSDI-8 & 200 & -90 & & & 500 & & & \\
\hline MMSDI-9 & 200 & -120 & & & 500 & & & \\
\hline MMSDI-10 & 150 & -60 & & & 50 & & & \\
\hline
\end{tabular}

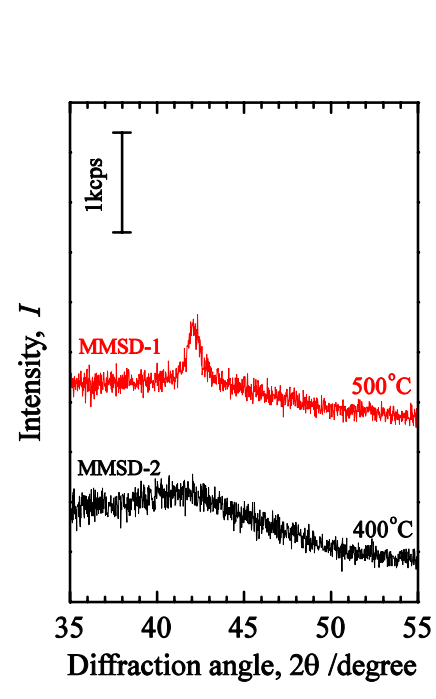

(a) without ion irradiation

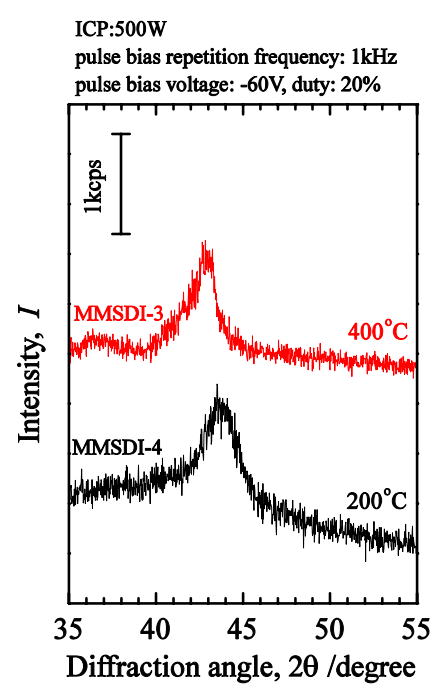

(b) with ion irradiation

Fig. 2. Room temperature X-ray diffraction profiles of deposited films formed at the different substrate temperatures.

(a) without ion irradiation and (b) with ion irradiation.

In order to clarify the ion-irradiation effects on the crystallization temperature, the plasma density was measured by double probe under various plasma conditions. The ion fluence rate for irradiation is correlated directly with the plasma density in case of the constant pulse bias voltage. Fig. 3 shows the relationship between RF power and plasma density in the ICP measured with double probe. The plasma density in ICP is shown as a function of RF power. From this figure we can see that the plasma density increases roughly proportionally to the RF power. Therefore, the ion fluence increases proportionally to RF power, if the ion-irradiation condition is constant. Fig. 4 shows the XRD profiles of deposited films formed under the conditions of (a) the different RF powers for ICP with $-60 \mathrm{~V}$ of the constant pulse bias voltage and (b) the different pulse bias voltages with $500 \mathrm{~W}$ of the constant 
RF power. The every sample except MMSDI-9 in Fig. 4 has the peak around at $2 \theta=44^{\circ}$. From Fig. 4 (a) we can see that the peak-intensity increase is not proportional to the RF power, which is roughly proportional to the plasma density as shown in Fig.3. In addition the half-value width of the peak is almost constant against the RF power. This implies that the ion fluence rate, which is also proportional to the plasma density, would not contribute so much to the crystal growth, although the crystal orientation might be influenced by the ion fluence. On the other hand, the sample MMSDI-9, which was made with $-120 \mathrm{~V}$ of the pulse bias voltage, does not indicate any of typical XRD peaks of TiNi crystal as shown in Fig. 4 (b). It is considered that the ion-irradiation energy has an optimum value for crystallization. When a pulse bias voltage larger than $-90 \mathrm{~V}$ was applied to the substrate holder in the apparatus, the half-value width in the XRD spectra changed more clearly rather than the peak intensity. Comparing the XRD profile of sample MMSDI-4 with that of MMSDI-8, we can see that the peak becomes broader even for a slight bias-voltage increase of $30 \mathrm{~V}$. It is considered that the peak broadening comes from the strain increase in TiNi structure by ion collision. These results give suggestions that the too-high energy of ions would break the crystal structure or block the crystal growth, but the proper energy of ions would promote the crystal growth. Therefore, the simultaneous ion irradiation is considered to have some opposite effects, i.e. promoting and blocking the crystal growth at the same time. Which effect is more predominant will be determined by the combination of those parameters such as deposition rate, substrate temperature, ion fluence and ion energy.

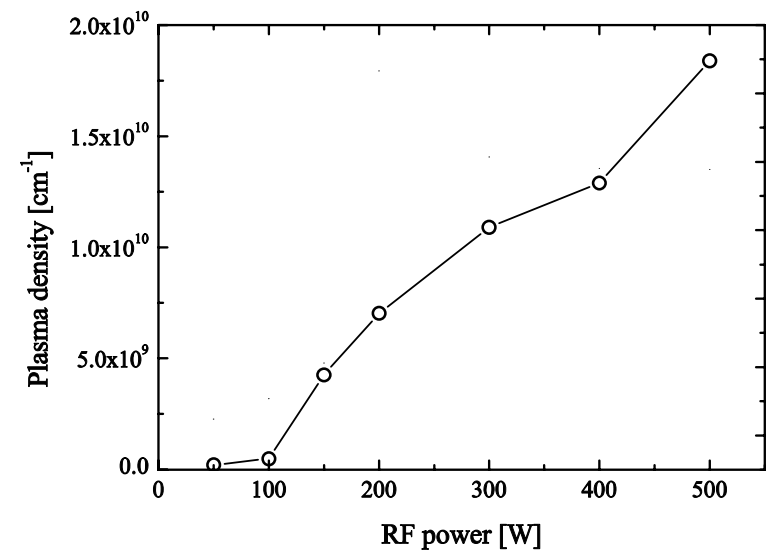

Fig. 3. Relationship between RF power and plasma density in the ICP measured with double probe.

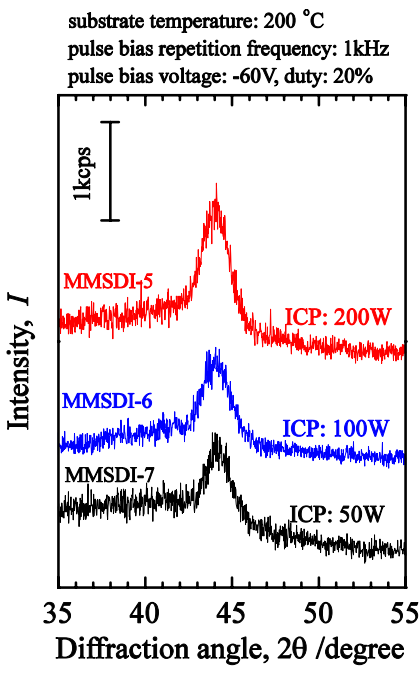

(a) RF power dependency

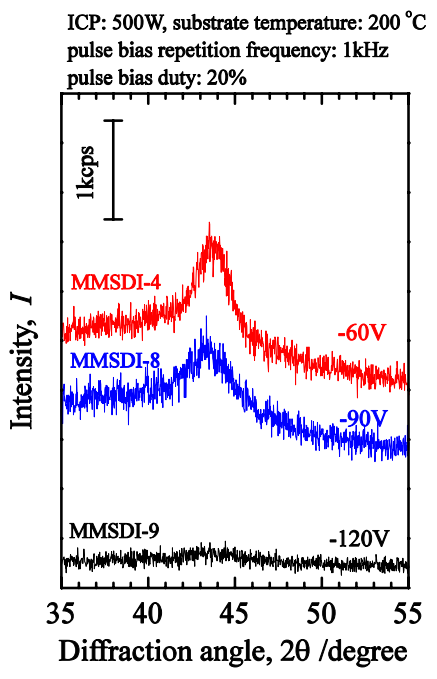

(b) pulse bias voltage dependency

Fig. 4. Room temperature X-ray diffraction profiles of deposited films formed under the conditions of (a) the different RF powers for ICP generation and (b) the different pulse bias voltages at $200{ }^{\circ} \mathrm{C}$ of substrate temperature. 
Then, we try to further lower the crystallization temperature. The lowest RF power for ICP, $50 \mathrm{~W}$, is selected among the RF powers with which we have confirmed crystallization, since the ion fluence rate, which is also proportional to the plasma density, would not contribute so much to the crystal growth, as mentioned above. This also helps for lowering the substrate temperature, because the ion irradiation energy that would cause heating the substrate can be lowered. Fig. 5 shows the XRD profile of the sample MMSDI-10 that was made at $150{ }^{\circ} \mathrm{C}$ of substrate temperature, compared with MMSDI-7 made at $200^{\circ} \mathrm{C}$.

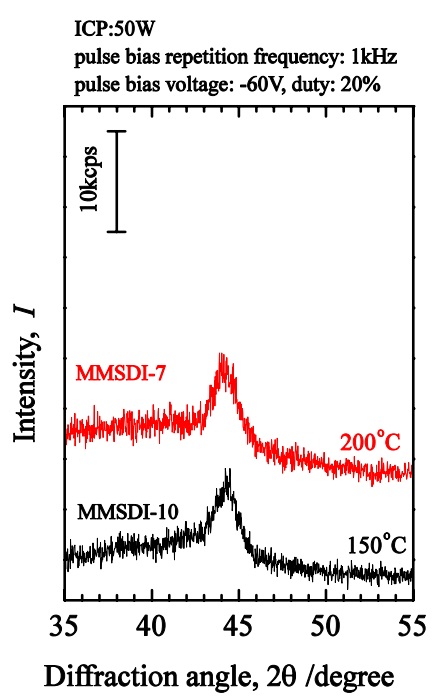

Fig. 5. Room temperature X-ray diffraction profiles of deposited films formed at $200{ }^{\circ} \mathrm{C}$ and $150{ }^{\circ} \mathrm{C}$ of substrate temperature.

The optimum bias-voltage $(-60 \mathrm{~V})$ for this experiment (after improving the bias-voltage feeder) is different from that $(-300 \mathrm{~V})$ for previous experiment $[7,8]$. However, the optimum ion energy is considered same. When considerable area of the ICP plasma contacts with the substrate holder or its feeder, the plasma potential of the ICP for ion irradiation also changes itself depending on the pulse bias voltage. The amount of the plasma potential variation depends on the ratio of the bias-voltage area to the grounded area that the plasma contacts with, too. Therefore, the bias-voltage for this experiment is closer to the exact ion energy in $\mathrm{eV}$.

Anyway, the ion energy can be controlled by changing the pulse bias voltage applied to the substrate, although the ion energy expressed in $\mathrm{eV}$ is not exactly equal to the pulse bias voltage in $\mathrm{V}$.

\section{Conclusion}

Up to now, we have been trying to lower the crystallization temperature of TiNi alloy. We developed a new apparatus as well as a new process for lowering the crystallization temperature by using ion irradiation, and realized the low temperature crystallization at $200{ }^{\circ} \mathrm{C}$ of substrate temperature [8]. This time, in order to further lower the crystallization temperature we have improved the bias-voltage introduction as well as optimizing the ion irradiation conditions. Resultantly, we have realized the low temperature crystallization at $150{ }^{\circ} \mathrm{C}$ of substrate temperature. In this paper we have also revealed some new details concerned with lowering the crystallization temperature by ion irradiation. The ion energy can be controlled by changing the pulse bias voltage applied to the substrate, although the ion energy expressed in $\mathrm{eV}$ is not exactly equal to the pulse bias voltage in $\mathrm{V}$. The exact value for the optimum ion energy in $\mathrm{eV}$ will be closer to the bias-voltage for the experiment after the feeder improvement.

\section{Acknowledgements}

The present study was partly supported by “Open Research Center” Project (2006-2010) for Private Universities: matching fund subsidy from MEXT and the second stage of the MEXT Knowledge Cluster Initiative Project -Hokuriku Innovation Cluster for Health Science- (2008-2012). YK, NS and ZY acknowledge the Grant-in-Aid for Basic Researches (C) (19560710, 19560323 and 21560727) from MEXT. 


\section{References}

[1] K.R.C. Gisser, J.D. Bush, A.D. Johnson, A.B. Ellis, Oriented nickel-titanium shape memory alloy films prepared by annealing during deposition, Appl. Phys. Lett. 61 _14._1992. .

[2] Y. Yang, H.S. Jia, Z.F. Zhang, H.M. Shen, A. Hu, Y.N. Wang, Transformations in sputter-deposited thick films of NiTi shape memory alloy, Materials Letters 22_1995.137.

[3] J.D. Bush, A.D. Johnson, C.H. Lee, D.A. Stevenson, Shape memory properties in Ni-Ti sputter-deposited films, J. Appl. Phys. 68 _12._1990.6224.

[4] J. L. Seguin, M. Bendahan, A. Isalgue, V. Esteve-Cano, H. Carchano, V. Torra, Low temperature crystallized Ti-rich NiTi shape memory alloy films for microactuators, Sensor and Actuators 74, (1999) 65-69.

[5] H. Funakubo, Shape-Memory Alloys, Gordon and Breach, New York, 1984.

[6] S. Miyazaki, A. Ishida, Martensitic transformation and shape memory behavior in sputter-deposited TiNibase thin films, Mater. Sci. Eng. A Vol. 273-275 (1999) 106-133

[7] N. Ikenaga, Y. Kishi, Z. Yajima and N. Sakudo, Influence of substrate temperature on texture for deposited TiNi films, Advances in Science and Technology Vol. 59 (2008) pp 30-34

[8]Y. Kishi, N. Ikenaga, N. Sakudo and Z. Yajima, Transformation behavior of low temperature crystallized TiNi shape memory alloy films, in this proceeding of ESOMAT 2009 